\title{
Adrenergic Neural Connections between the Bilateral Supraoptic Nuclei of the Rat Hypothalamus
}

\author{
Hiromi Tsushima, Mayumi Mori and Tomohiro Matsuda ${ }^{\#}$ \\ Department of Pharmacology, Nagoya City University Medical School, Kawasumi, Mizuho-ku, Nagoya 467, Japan \\ Received October 30, 1995 Accepted March 1, 1996
}

\begin{abstract}
Our previous study has demonstrated that unilateral microinjection of norepinephrine (NE) into the right supraoptic nucleus (SON) of anesthetized hydrated rats elicited dose-dependent decreases in the urine outflow rate. This was antagonized by pretreatment with phenoxybenzamine (an $\alpha$-antagonist) and timolol (a $\beta$-antagonist) in the same SON. In the present study, we examined the effects of NE, microinjected into the right, left and bilateral SON, on the urine outflow rate in order to investigate neural connections between the bilateral SON. NE administered by those three routes dose-dependently decreased the urine outflow rate. The order for the antidiuretic potency was as follows: the effect elicited by the intrabilateral-SON microinjection $>$ the intra-left-SON microinjection $=$ the intra-right-SON microinjection. The antidiuresis of NE microinjected into the right SON was inhibited by an electrolytic left-SON lesion and by pretreatment with phenoxybenzamine $(20 \mathrm{nmol})$ and timolol $(100 \mathrm{nmol})$, but not by atropine $(300 \mathrm{nmol})$ in the left SON. These findings suggest adrenergic neural connections from the right to left SON, contributing to the regulation of urine production. Furthermore, there is a possibility that stimulation of endogenously-released NE in the bilateral SON is amplified through these neurons and elicits more potent effects than those produced in either the right or left nucleus.
\end{abstract}

Keywords: Supraoptic nucleus, Hypothalamus, Norepinephrine, Adrenergic neuron, Antidiuresis

The hypothalamic supraoptic (SON) and paraventricular nuclei (PVN) synthesize the antidiuretic hormone vasopressin (AVP), which plays an important role in regulating the osmotic pressure of the body fluid through changes in urine volume. Norepinephrine (NE) and the other adrenoceptor agonists, when unilaterally microinjected into the nuclei, have been already shown to decrease urine production resulting from an increase in plasma AVP level mediated through $\alpha_{1}$-adrenoceptors $(1-7)$. An increase in AVP release induced by injection of NE into the SON and PVN is specific and the highest among the ones induced by injection of NE into the other hypothalamic nuclei (3). $\alpha_{2}$-Adrenoceptors in the nuclei do not appear to be involved in the regulation of AVP release (4). Noradrenergic neurons project to the SON and PVN from the $A_{1}$ and/or $A_{2}$ region (8-13) and consist of synapses with AVP-containing neurons in the nuclei (14). Electric stimulation of the $A_{1}$ or $A_{2}$ region excites the neuron and promotes AVP release $(9-11)$.

The SON and PVN exist in the right and left sides.
Neural connections between the contralateral and ipsilateral nuclei are suggested by electrophysiological and anatomical studies $(13,15-19)$. The four nuclei may cooperatively function for the regulation under physiological conditions. However, neither their functional interactions nor the neurotransmitters involved in the connections are clear.

In the present study, we focussed on the neural connections to the left SON from the right SON, and we investigated the effects of $\mathrm{NE}$, microinjected bilaterally into the SON, on the urine outflow rate; the data were compared with the results from unilateral, right and left SON microinjection. In addition, we examined the influence of an electrolytic left-SON lesion and the effect of pretreatment with antagonists in the left SON on NE-induced antidiuresis in the contralateral SON. The urine outflow rate is constant for several hours, and it is possible to continuously observe any changes. The objectives of the present study are to obtain pharmacological evidence for neural connections between the bilateral SON and to identify the neurotransmitters involved. 


\section{MATERIALS AND METHODS}

\section{Surgical procedure}

One day before the experiments, male Wistar rats (280-350 g, 9- to 10-week-old; Kitayama Labes Co., Ina) were starved from 4 p.m., but allowed free access to water. On the day of the experiments, the rats were orally administered a volume of $5 \mathrm{ml} / 100 \mathrm{~g}$ body weight of tap water and, $45 \mathrm{~min}$ later, were also given the same volume of $12 \%$ ethanol for anesthesia. After cannulation into the urinary bladder, jugular vein and trachea, the rats were placed in a stereotaxic frame (Takahashi Co., Tokyo).

\section{Measurement of urine outflow rate}

During the experiments, Locke's solution containing $3 \%$ ethanol was continuously infused at a rate of 0.10 $\mathrm{ml} / \mathrm{min}$ through the jugular vein cannula. Administration of water and ethanol permits a measurably constant rate of urine outflow for approximately $6 \mathrm{hr}$. Drops of urine from the bladder cannula were counted with a photoelectric drop counter (DCT 102; Unique Medical, Inc., Tokyo) every $10 \mathrm{~min}$. Changes in the urine outflow rate after drug administration were presented as a percentage of the control level (the urine outflow rate before drug administration).

\section{Administration of drugs}

After the urine outflow rate became constant for at least $20 \mathrm{~min}, 1 \mu \mathrm{l}$ of a drug solution (NE: $0.5-80 \mathrm{nmol}$, phenoxybenzamine: $20 \mathrm{nmol}$, timolol: $100 \mathrm{nmol}$, atropine: $300 \mathrm{nmol}$ ) was microinjected into the right, left or bilateral SON at a rate of $0.35 \mu \mathrm{l} / \mathrm{min}$ through the cannula $(0.25 \mathrm{~mm}$ in outer diameter) connected to a microsyringe. For the bilateral administration, the cannula in the right SON had to be diagonally inserted at an angle of 12 degrees from the vertical line because of the design of the stereotaxic frame. The coordinate for the SON $(6.3 \mathrm{~mm}$ anterior from lambda, $1.3 \mathrm{~mm}$ lateral from the midline and $8.9 \mathrm{~mm}$ ventral from the dural surface) was determined according to the atlas of König and Klippel (20). Three injections of the drugs were, at the most, performed in one animal.

\section{Experiment for antagonists and lesion}

We investigated the influences of antagonists microinjected into the left SON or left-SON lesion on antidiuresis induced by intra-right-SON microinjection of NE (20 $\mathrm{nmol}$ ) in one rat. At first, the antidiuresis was confirmed after microinjection of NE into the right SON. When the decrease in the urine outflow rate recovered to the control level, one of the antagonists was microinjected into the left SON or a lesion was made by a lesion generator system (Radionics RFG-4A Research RF Lesion Generator
System; Radionics, Inc., Burlington, MA, USA). The electrode was $0.25 \mathrm{~mm}$ in outer diameter with an exposed tip 0.25 -mm-long. The tissue temperature was raised to $55^{\circ} \mathrm{C}$ for $60 \mathrm{sec}$. After $40 \mathrm{~min}$, the second microinjection of $\mathrm{NE}$ into the right SON was performed in the same rat.

\section{Histological identification}

When the experiments were finished, the brain was removed after decapitation and coronal $30-\mu \mathrm{m}$ sections were cut using a cryostat (Tissue-Tek II; Miles, Inc., Elkhart, IN, USA). The injection sites were verified under a microscope. Figure 1 represents the injection sites for bilateral microinjection of $5 \mathrm{nmol} \mathrm{NE}$ into the SON. The size of the lesion was $1.6 \mathrm{~mm}$ in height, $1.2 \mathrm{~mm}$ in width and $1.5 \mathrm{~mm}$ in length. Rats with incorrectly localized microinjection sites and lesions were omitted from the results.

\section{Statistics}

All data are expressed as the mean \pm S.E. One-way ANOVA followed by Scheffe's test was used for statistical analysis between two means at one time point. A significant difference was assumed when the $P$ value was less than 0.05 .
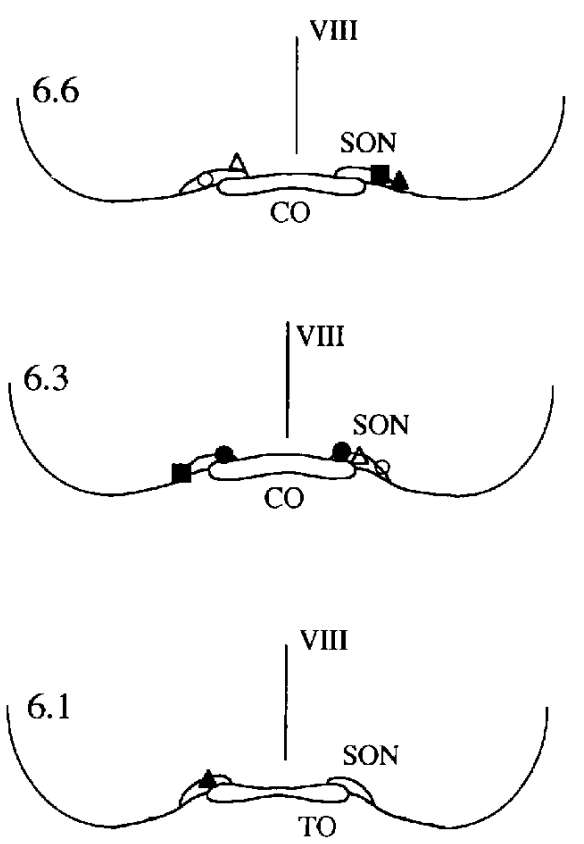

Fig. 1. Schematic representation of bilateral microinjection sites in the right and left SON for $5 \mathrm{nmol}$ NE. Each of the same symbols in the right and left SON indicate simultaneous microinjection sites into the bilateral nuclei of one rat. The numbers in the upper lefthand corner of each panel denote millimeters anterior to lambda. Antidiuretic effects of these experiments are shown in Fig. 2. SON: supraoptic nucleus, VIII: third ventricle, $\mathrm{CO}$ : chiasma opticum, TO: tractus opticus. 


\section{Drugs}

The following drugs were used: l-norepinephrine bitartrate (Sigma Chemical Co., St. Louis, MO, USA), phenoxybenzamine hydrochloride (Nacalai Tesque, Kyoto), $d$-timolol hydrochloride (Sankyo Co., Tokyo) and atropine sulfate (Iwaki Co., Tokyo). The other chemicals used were of the analytical grade available.

\section{RESULTS}

\section{Effects of $N E$ on the urine outflow rate}

Microinjection of $\mathrm{NE}$ at $5-80 \mathrm{nmol}$ into the right SON caused antidiuresis in a dose-dependent manner, as shown in Fig. 2. The antidiuresis was slow in onset; the time to the maximum response being $30 \mathrm{~min}$. The duration was approximately $70 \mathrm{~min}$. NE, microinjected into the left SON, produced antidiuresis with a similar timecourse to that in the right SON. The potency for the antidiuresis elicited in the left SON appeared to be almost the same as that in the right SON. Simultaneous microinjection of $\mathrm{NE}$ into the bilateral SON induced the most potent antidiuresis among the three administration routes. $\mathrm{NE}$ at $2 \mathrm{nmol}$, microinjected into the right or left SON, did not show any effects on the urine outflow rate. In contrast, microinjection of this dose into both the right and left SON elicited a potent decrease in the rate. The dose-response curve for the bilateral SON microinjection of NE significantly shifted to the left of the others (Fig. 2). The $E D_{50}$ values for the antidiuresis in the right, left and bilateral SON were found to be 15,13 and 1.3

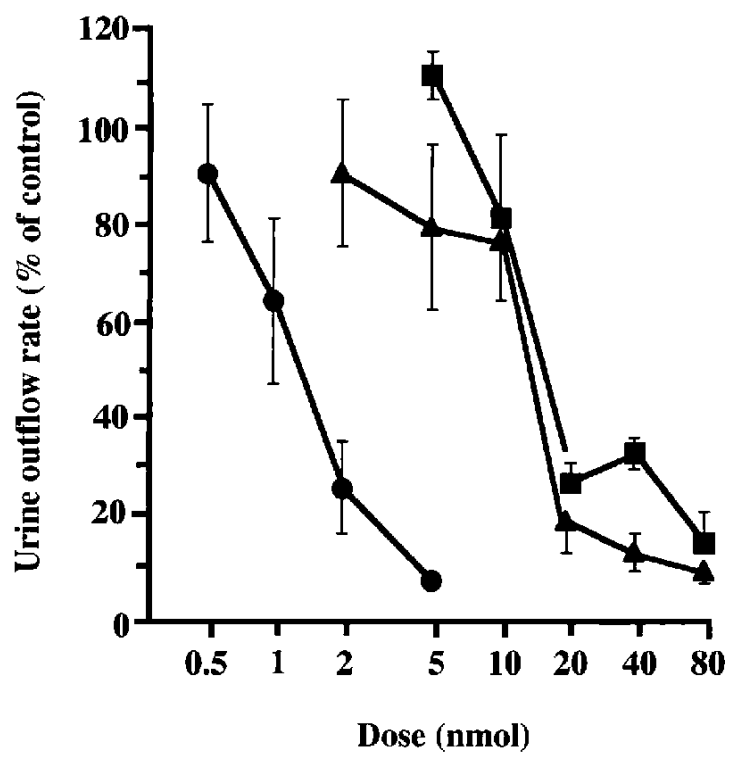

Fig. 2. Dose-response curves for antidiuresis by microinjection of NE into the bilateral (O), left (A) and right ( $\square$ ) SON. Abscissa: dose in nmol. Ordinate: maximum decrease in the urine outflow rate 30 min after microinjection of NE, expressed as a percentage of the control level $(117 \pm 1, \mu 1 / \mathrm{min}, \mathrm{n}=91)$. All values are means \pm S.E. for 4-11 experiments.

(in each side) nmol, respectively, from the dose-response curves in Fig. 2. Microinjection of NE at $20 \mathrm{nmol}$ was performed into the sites at a distance of $1 \mathrm{~mm}$ above the right $\mathrm{SON}, 1 \mathrm{~mm}$ rostral and lateral from the SON, to examine diffusion of the microinjected drug. None of the

Table 1. Effect of vehicle, $\mathrm{NE}$ and $\mathrm{KCl}$ microinjected into the SON and/or outside the SON on urine outflow rate

\begin{tabular}{llrrrrrrr}
\hline & & \multicolumn{7}{c}{ Urine outflow rate (\% of control) } \\
\cline { 3 - 9 } Drug & Site & & \multicolumn{7}{c}{ Time after microinjection (min) } \\
\cline { 3 - 9 } & & 0 & 10 & 20 & 30 & 40 & 50 & 60 \\
Vehicle & both SON & 100 & $95 \pm 6$ & $87 \pm 7$ & $97 \pm 2$ & $97 \pm 4$ & $101 \pm 6$ & $101 \pm 6$ \\
Vehicle & right SON & 100 & $104 \pm 2$ & $101 \pm 3$ & $100 \pm 3$ & $95 \pm 4$ & $92 \pm 4$ & $91 \pm 5$ \\
$\mathrm{NE}$ & above & 100 & $100 \pm 5$ & $108 \pm 11$ & $96 \pm 5$ & $100 \pm 3$ & $99 \pm 3$ & $99 \pm 2$ \\
& right SON & 100 & $81 \pm 13$ & $18 \pm 6$ & $13 \pm 4$ & $29 \pm 11$ & $61 \pm 18$ & $87 \pm 26$ \\
$\mathrm{NE}$ & anterior & 100 & $121 \pm 15$ & $99 \pm 4$ & $92 \pm 4$ & $97 \pm 11$ & $102 \pm 15$ & \\
& right SON & 100 & $121 \pm 13$ & $22 \pm 8$ & $14 \pm 6$ & $20 \pm 4$ & $62 \pm 17$ & $118 \pm 16$ \\
$\mathrm{NE}$ & lateral & 100 & $117 \pm 4$ & $104 \pm 6$ & $108 \pm 6$ & $110 \pm 4$ & & \\
& right SON & 100 & $106 \pm 16$ & $25 \pm 7$ & $22 \pm 5$ & $28 \pm 3$ & $80 \pm 5$ & $129 \pm 8$ \\
$\mathrm{KCl}$ & right SON & 100 & $97 \pm 11$ & $17 \pm 4$ & $11 \pm 2$ & $21 \pm 5$ & $56 \pm 11$ & $103 \pm 12$ \\
$\mathrm{KCl}$ & left SON & 100 & $87 \pm 4$ & $14 \pm 3$ & $24 \pm 10$ & $34 \pm 14$ & $49 \pm 14$ & $71 \pm 9$ \\
\hline
\end{tabular}

Data represent means \pm S.E. of 3-10 experiments. Vehicle ( $1 \mu 1$, saline) was microinjected into the bilateral SON or the right SON. NE at $20 \mathrm{nmol}$ was microinjected into the sites $1 \mathrm{~mm}$ above the right SON, $1 \mathrm{~mm}$ anterior or lateral to the right SON, before or after the same dose of NE was administered into the right SON of the same rats. $\mathrm{KCl}$ at $800 \mathrm{nmol}$ was administered in the right or left SON. The control urine outflow rate was $92 \pm 8 \mu 1 / \min (n=14)$ for the vehicle, $92 \pm 8 \mu 1 / \mathrm{min}(\mathrm{n}=22)$ for $\mathrm{NE}$ and $118 \pm 6 \mu \mathrm{l} / \mathrm{min}(\mathrm{n}=15)$ for $\mathrm{KCl}$. 
NE administered into the three sites outside the nucleus showed any significant effects on the urine outflow rate (Table 1).

The antidiuretic response for $\mathrm{KCl}$ at $800 \mathrm{nmol}$ in the left SON was not different from that in the right SON (Table 1). We have already reported that $\mathrm{KCl}$ as a depolarizing agent elicited dose-dependent antidiuresis at a dosage range of $200 \mathrm{nmol}$ to $1.6 \mu \mathrm{mol}$ in the right SON using the same methods (5).

The vehicle (saline), microinjected into the right and bilateral SON, did not change the urine outflow rate (Table 1).

\section{Microinjection of NE into the right SON of electrolytic left-SON-lesioned rats}

The difference in antidiuretic potency of NE administered bilaterally and unilaterally suggests the existence of neural connections between the two SONs. In order to verify this hypothesis, we investigated the effect on the urine outflow rate of NE microinjected into the right SON after an electrolytic left-SON lesion.

As shown in Fig. 3A, at 30-70 min after injection, there were statistically significant differences in the urine outflow rates before and after the lesion. The lesion diminished antidiuresis induced by the right SON microinjection of NE. However, a weak decrease in the urine outflow rate still remained. The lesion itself caused a temporary decrease in the urine outflow rate in two out of five rats $(71 \%$ at $10 \mathrm{~min}, 68 \%$ at $20 \mathrm{~min}, 81 \%$ at 30 $\min , 84 \%$ of the control level at $40 \mathrm{~min}$ after the injection; control level: $82 \mu \mathrm{l} / \mathrm{min}, \mathrm{n}=2$ ). For this reason, the second injection of NE into the right SON was carried out $40 \mathrm{~min}$ after the lesion.

As controls, microinjection of $\mathrm{NE}$ at $20 \mathrm{nmol}$ was performed in five rats lesioned at a site $3 \mathrm{~mm}$ above the left SON (sham-lesioned rats); and in six rats, the electrode was inserted into the left SON without an electric current (non-lesioned rats). In both experiments, antidiureses induced by the first and second microinjection of NE at 20 nmol into the right SON were equivalent to each other (Fig. 3: B and C).

\section{Microinjection of NE into the right SON of rats pretreated with adrenoceptor and muscarinic antagonists in the left SON}

We investigated the involvement of adrenoceptors and muscarinic receptors in the left SON in the antidiuresis induced by microinjection of NE into the right SON.

Pretreatment with atropine at $300 \mathrm{nmol}$ (a muscarinic antagonist) in the left SON was performed 40 min before microinjection of NE into the right SON. Atropine, which by itself did not influence the urine outflow rate, showed no change in the effect induced by the right SON
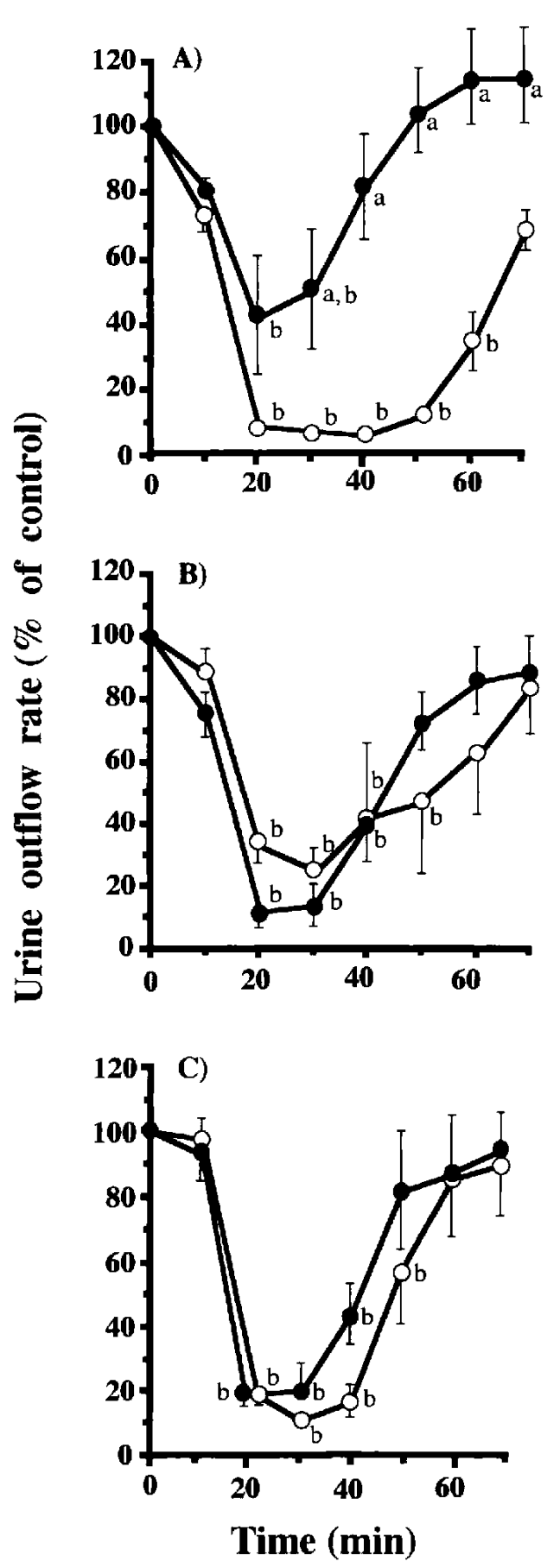

Fig. 3. Effect of electrolytic left-SON lesion on antidiuresis by microinjection of NE into the right SON. $\bigcirc$ and $D$ indicate the urine outflow rate after microinjection of $\mathrm{NE}$ at $20 \mathrm{nmol}$ into the right SON without and with the treatment, respectively, ( $A$ : leftSON lesion by an electric current, $n=5 ; B$ : sham lesion of the site 3 $\mathrm{mm}$ above the left SON by an electric current, $n=5 ; C$ : the same procedure as panel $A$ without an electric current, $n=6$ ). Time between the lesion and the second injection of NE was $40 \mathrm{~min}$. The control urine outflow rate was $107 \pm 7 \mu \mathrm{l} / \mathrm{min} .{ }^{\mathrm{a}} \mathrm{P}<0.05$ vs $\bigcirc$ at each corresponding time point. ${ }^{b} \mathrm{P}<0.05$ vs the values at each corresponding time point after microinjection of vehicle into the right SON (Table 1). 

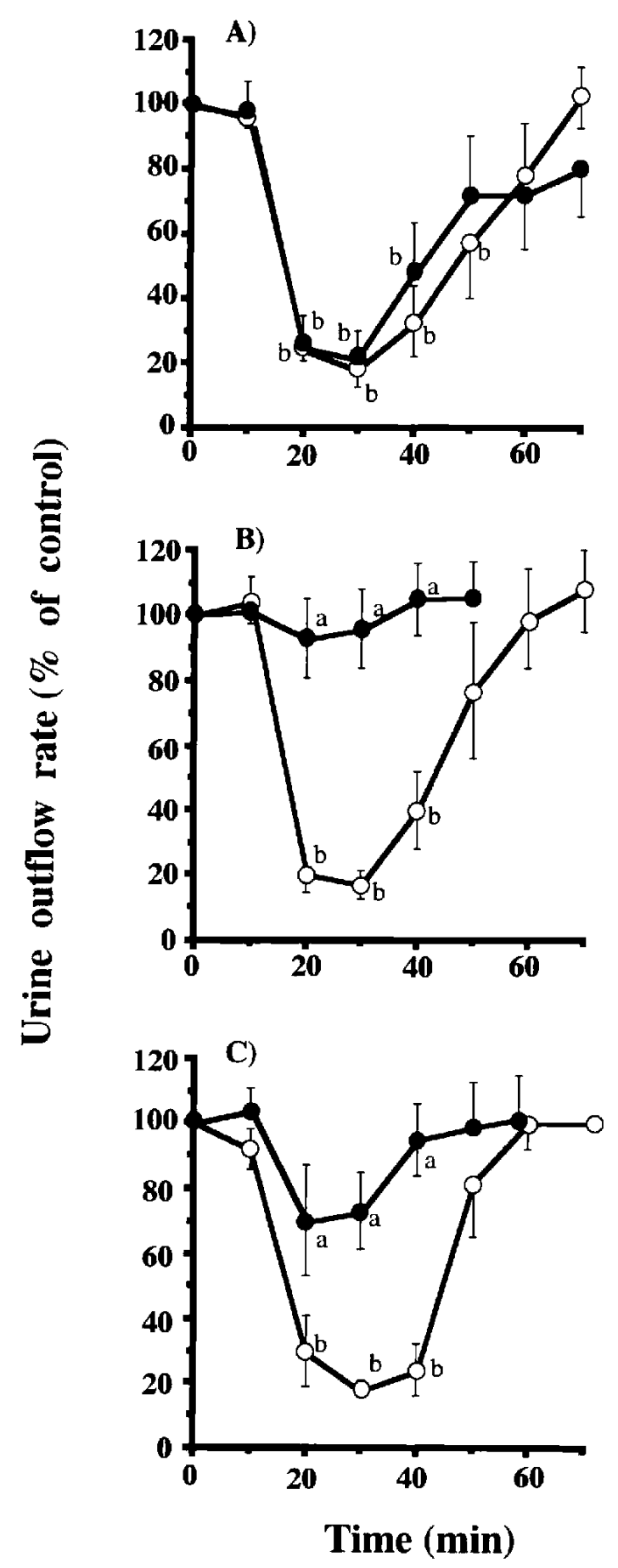

Fig. 4. Effect of muscarinic and adrenoceptor antagonists on antidiuresis by microinjection of NE into the right SON. $O$ and $O$ indicate the urine outflow rate after microinjection of $\mathrm{NE}$ at $20 \mathrm{nmol}$ into the right $\mathrm{SON}$ in the absence and presence of the antagonists, respectively, (A: $300 \mathrm{nmol}$ atropine, $\mathbf{n}=5$; B: $20 \mathrm{nmol}$ phenoxybenzamine, $n=5 ; C$ : 100 nmol timolol, $n=4$ ). The pretreatment with the antagonists in the left SON was $40 \mathrm{~min}$ before the second administration of NE into the contralateral SON. The control urine outflow rate was $107 \pm 9 \mu \mathrm{l} / \mathrm{min}$. ${ }^{\mathrm{a}} \mathrm{P}<0.05$ vs $\bigcirc$ at each corresponding time point. ${ }^{b} \mathrm{P}<0.05$ vs the values at the corresponding time point after microinjection of vehicle into the right SON (Table 1). microinjection of NE (Fig. 4A).

On the other hand, pretreatment of the left SON with phenoxybenzamine at $20 \mathrm{nmol}$ (an $\alpha$-adrenoceptor antagonist) or timolol at $100 \mathrm{nmol}$ (a $\beta$-adrenoceptor antagonist) at $40 \mathrm{~min}$ before the administration of NE inhibited the effects of NE microinjected into the contralateral SON (Fig. 4: B and C). The inhibitory effect of timolol was partial. Microinjection of phenoxybenzamine into the left SON did not change the urine outflow rate, but that of timolol alone in the left SON slightly increased it at $40 \mathrm{~min}$ after the administration $(143 \pm 5 \%$ of the control level at $40 \mathrm{~min}$ after the administration, control level: $115 \pm 26 \mu \mathrm{l} / \mathrm{min}, \mathrm{n}=4$ ).

\section{DISCUSSION}

In the present study, we demonstrated that all microinjections of NE into the right, left and bilateral SON elicited dose-dependent antidiuresis and that the order for antidiuretic potency was as follows: the effect elicited by the intra-bilateral-SON microinjection $>$ the intra-leftSON microinjection $=$ the intra-right-SON microinjection. The antidiuresis is suggestd to result from increased secretion of AVP, because microinjection of NE into the SON promotes AVP release $(3,4)$ and does not change the blood pressure (5). The most powerful effect elicited by intra-bilateral-SON injection of NE suggests neural contacts between the right and left SON. If two nuclei are independent, a drug simultaneously administered to both of them should produce the sum of the each effects elicited in the two nuclei. The presence of neural connections between the bilateral SON were also supported by the result that an electrolytic lesion of the left SON and the preteatments with the adrenoceptor antagonists in the left SON diminished the NE-induced effect elicited in the contralateral SON. However, this experiment merely shows neural connections from the right to the left SON, not reciprocal connections between the two nuclei. The inhibition by electrical lesion was partial and a weak, but significant, antidiuresis remained. Therefore, stimulation of NE applied to the right SON seems to be partly transferred to the contralateral SON, resulting in antidiuresis.

There is no anatomical evidence for neural connections between the right and left SON; however, electrophysilolgical studies show contacts between them. Takano et al. (19) showed that one-third of the AVPcontaining neurons tested in the SON are excited by contralateral-SON electric stimulation. This is consistent with our present result. However, the other one-third were inhibited by it. On the other hand, the majority of oxytocin-containing neurons in the nuclei are excited by contralateral-SON electric stimulation. Moos and coworkers $(15,16)$ demonstrated synchronized bursting 
activities of oxytocin-containing neurons in the bilateral SON and PVN to suckling stimuli and intra-SON injection of oxytocin. The electrophysiological studies can not elucidate functional interactions and the receptors and/or transmitters involved in neural connections.

To identify neurotransmitters in these neural connections, we pretreated the left SON with the antagonists before administration of NE in the contralateral SON. The $\alpha$ - and $\beta$-antagonists, but not the muscarinic antagonist, inhibited the NE-induced effect. This suggests involvement of adrenoceptors in the left SON. In other words, administration of NE into the right SON stimulates $\mathrm{NE} /$ epinephrine release in the contralateral SON. This, at least in part, accounts for antidiuresis induced by intraright-SON injection of NE. Both stimulation of $\alpha$ - and $\beta$-receptors in the nucleus decreased urine outflow rates to the same extents (5), although the mechanisms are suggested to be different $(3-5,21)$. The inhibition by phenoxybenzamine was almost complete and seemed to be slightly greater than that by timolol. Therefore, $\alpha$ adrenoceptors may function more powerfully than $\beta$ adrenoceptors. On the other hand, phenoxybenzamine alone did not influence the urine outflow rate, and timolol alone elicited diuresis. This suggests that $\beta$-adrenoceptors regulate urine production in the control condition. After all, adrenoceptors play an important role in the regulation of urine production. Also, the other neurotransmitters will contribute to the effect, since Takano et al. (19) conclude that neural connections between the bilateral SON are polysynaptic.

The pathway for these neural connections is not understood. In general, the commissura anterior, corpus callosum and commissura fornicis are the main tractus nervosi commissurales. Moos and Richard (16) concluded that the supraventricular gray commissura is important for interconnection of oxytocin-containing neurons in the SON, because synchronization of oxytocin-containing neurons in the bilateral SON disappears after interhemisphere section (including the supraventricular gray commissura and the corpus callosum), but persists after superficial interhemisphere section limited to the corpus callosum. Therefore, the supraventricular gray commissura is a possible pathway for interconnections of bilateral AVP-containing neurons in the SON. Also, the other contacts between the bilateral SON through the medulla oblongata, pons and so on are speculated. Moreover, there is a possibility that increased plasma concentration of AVP, after stimulation of the right SON, activated the contralateral SON through the other nuclei.

Nuclei in the thalamus, the hypothalamus, medulla obIongata and so on exist in both the right and left sides. There is accumulating evidence that interconnections between bilateral nuclei are important for their functions
$(22-30)$. In this study using a pharmacological technique, we demonstrated that adrenergic neural connections from the right SON to the contralateral SON are involved in the regulation of urine production by NE administered in the right SON.

\section{REFERENCES}

1 Day TA, Randle ICR and Renaud LP: Opposing $\alpha$ - and $\beta$ adrenergic mechanisms mediate dose-dependent actions of noradrenaline on supraoptic vasopressin neurons in vivo. Brain Res 358, 171-179 (1985)

2 Leibowitz SF: Impact of brain monoamines and neuropeptides on vasopressin release. In Vasopressin: Cellular and Integrative Functions, Edited by Cowley AW Jr, Liard J-F and Ausiello DA, pp 379-388, Raven Press, New York (1988)

3 Leibowitz SF, Eidelman D, Suh JS, Diaz S and Sladek CD: Mapping study of noradrenergic stimulation of vasopressin release. Exp Neurol 110, 298-305 (1990)

4 Qadri F, Culman J, Veltmar A, Maas K, Rascher W and Unger $\mathrm{T}$ : Angiotensin II-induced vasopressin release is mediated through alpha-1 adrenoceptors and angiotensin II AT1 receptors in the supraoptic nucleus. J Pharmacol Exp Ther 267, $567-574$ (1993)

5 Tsushima $\mathbf{H}$, Mori $\mathbf{M}$ and Matsuda T: Antidiuretic effects of alpha- and beta-adrenoceptor agonists microinjected into the hypothalamic supraoptic nucleus in a water-loaded and ethanol-anesthetized rat. Jpn J Pharmacol 39, 365-374 (1985)

6 Tsushima H, Mori $M$ and Matsuda T: Antidiuretic effects of alpha- and beta-adrenoceptor agonists microinjected into the hypothalamic paraventricular nucleus in a water-loaded and ethanol-anesthetized rat. Jpn J Pharmacol 40, 319-328 (1986)

7 Veltmar A, Culman J, Qadri F, Rascher W and Unger T: Involvement of adrenergic and angiotensinergic receptors in the paraventricular nucleus in the angiotensin II-induced vasopressin release. J Pharmacol Exp Ther 263, 1253-1260 (1992)

8 Davis BJ, Blair ML, Sladek JR Jr and Sladek CD: Effects of lesions of hypothalamic catecholamines on blood pressure, fluid balance, vasopressin and renin in the rat. Brain Res 405, $1-15$ (1987)

9 Day TA, Ferguson AV and Renaud LP: Facilitatory influence of noradrenergic afferents on the excitability of rat paraventricular nucleus neurosecretory cells. J Physiol (Lond) 355, 237-249 (1984)

10 Day TA and Renaud LP: Electrophysiological evidence that noradrenergic selectively facilitate the activity of supraoptic vasopressin neurons. Brain Res 303, 233-240 (1984)

11 Day TA and Sibbald JR: Al cell group mediates solitary nucleus excitation of supraoptic vasopressin cells. Am J Physiol 257, R1020-R1026 (1989)

12 Sawchenko PE and Swanson LW: The organization of noradrenergic pathway from the brainstem to the paraventricular and supraoptic nuclei in the rat. Brain Res Rev 4, 275-325 (1982)

13 Silverman AJ, Hoffman DL and Zimmerman EA: The descending afferent connections of the paraventricular nucleus of the hypothalamus (PVN). Brain Res Bull 6, 47-61 (1981)

14 Nakada $\mathrm{H}$ and Nakai $Y$ : Electron microscopic examination of the catecholaminergic innervation of neurophysin- or vasopres- 
sin-containing neurons in the rat hypothalamus. Brain Res $\mathbf{3 6 1}$, 247-257 (1985)

15 Belin $\mathrm{V}$ and Moos F: Paired recordings from supraoptic and paraventricular oxytocin cells suckled rats: recruitment and synchronization. J Physiol (Lond) 377, 369-390 (1986)

16 Moos F and Richard P: Paraventricular and supraoptic oxytocin cells in rat are locally regulated by oxytocin and functionally related. J Physiol (Lond) 408, 1-18 (1989)

17 Saphier D and Feldman S: Electrophysiologic evidence for neural connections between the paraventricular nucleus and neurons of the supraoptic nucleus in the rat. Exp Neurol 89, 289-294 (1985)

18 Saphier D and Feldman S: Electrophysiology of supraopticoparaventricular nucleus connections in the rat. Exp Brain Res 69, 60-66 (1987)

19 Takano S, Negoro H, Honda K and Higuchi T: Electrophysiological evidence for neural connections between the supraoptic nuclei. Neurosci Lett 111, 122-126 (1990)

20 König JFR and Klippel RA: The Rat Brain, A Stereotaxic Atlas of the Forebrain and Lower Parts of the Brain Stem. Williams and Wilkins Co, Baltimore (1963)

21 Tsushima $H$, Fujimoto $S$ and Matsuda T: Effects of $\hat{\beta}_{1}$ - and $\hat{\gamma}_{2}$-adrenoceptor agonists applied into the hypothalamic paraventricular nuclei of spontaneously hypertensive rats on urine production. Jpn J Pharmacol 64, $201-207$ (1994)

22 Alexander N, Kaneda N, Ishii A, Mogi M, Harada M and Nagatsu T: Right-left asymmetry of tyrosine hydroxylase in rat median eminence: influence of arterial baroreflex nerves. Brain Res 523, 195- 198 (1990)
23 Catelli JM, Giakas WJ and Sved AF: GABAergic mechanisms in nucleus tractus solitarius alter blood pressure and vasopressin release. Brain Res 403, 279-289 (1987)

24 Dewar D, Jenner P and Marsden CD: Behavioral effects in rats of unilateral and bilateral injections of opiate receptor agonists into the globus pallidus. Neuroscience 15, 41-46 (1985)

25 Ishii $\mathrm{K}$ and Kato $\mathrm{T}$ : Increase of dopamine turnover in bilateral striata after unilateral injection of haloperidol into substantia nigra of unrestrained rats. Brain Res 359, 260-266 (1985)

26 Leviel V, Chesselet MF, Glowinski $\mathbf{J}$ and Chéramy A: Involvement of the thalamus in the asymmetric effects of unilateral sensory stimuli on the two nigrostriatal dopaminergic pathways in the cat. Brain Res 223, 257-272 (1991)

27 Nieoullon A, Chéramy A and Glowinski J: Nigral and striatal dopamine release under sensory stimuli. Nature 269, 340-342 (1977)

28 Nieoullon A, Chéramy A and Glowinski J: Interdependence of the nigrostriatal dopaminergic systems on the two sides of the brain in the cat. Science 198, 416-418 (1977)

29 Romo R, Chéramy A, Godeheu G and Glowinski J: Distinct commissural pathways are involved in the enhanced release of dopamine induced in the contralateral caudate nucleus and substantia nigra by unilateral application of GABA in the cat thalamic motor nuclei. Brain Res 308, 43- 52 (1984)

30 Wilson R, Kamata K, Wightman RM and Rebec G: Unilateral, intranigral infusions of amphetamine produce differential, bilateral changes in unit activity and extracellular levels of ascorbate in the neostriatum of the rat. Brain Res 384, 342-347 (1986) 